\title{
A Novel three-phase unbalance Control Strategy of SST under Passive Microgrid
}

\author{
Ren-jie Ding, Caiqi-Zhou, Yu-wei Zhao \\ Department of Electrical Engineering, \\ Tsinghua University \\ Beijing, China \\ zhoucq10@mails.tsinghua.edu.cn
}

\begin{abstract}
Three-phase unbalance control strategy is used to improve the system performance when three-phase unbalance load or fault happens. However, when the distributed generation selected as the master in the microgrid stop working, due to the loss of system voltage direction, the traditional control strategy is no longer applicable. In order to maintain the stable operation of the SST (Solid State Transformer) under passive microgrid model when threephase unbalances occur in the microgrid, the paper came up a novel balance control strategy under passive microgrid model. The control strategy is based on the voltage and current double-loop control strategy, and uses a negative sequence current module to calculation the negative sequence reference current, prevent three-phase unbalance from entering into distribution grid side and improve the control performance of the SST. The simulation results have proved the validity and efficiency of the three-phase unbalance control strategy.
\end{abstract}

Keywords-Three-phase umbalance control strategy; solid state transformer; passive microgrid; negative-sequence current calculation module

\section{INTRODUCTION}

The distributed generation is able to make effective use of solar energy, wind power and other clear energies to reduce environment pollution, improve power supply stability and flexibility ${ }^{[1,2]}$. In order to effectively implement the distributed generation, the North Carolina State University puts forward a new intelligent microgrid model, the FREEDM (Future Rrenewable Electric Energy Delivery and Management) ${ }^{[3-5]}$. In the FREEDM model, a number of new electronics are introduced to replace original traditional mechanical equipment or electromagnetic equipment. The core equipment SST(Solid State Transformer), due to its effective reduction in transformer's volume, weight and noload loss, as well as its convenient control advantage, has attracted scholars' attention ${ }^{[6-8]}$.

The three-phase unbalance load and fault will cause the three-phase unbalance grid. For SST, its low-voltage AC system is not easy to maintain absolute three-phase balance among three phases. In other words, it may easily have unbalance issues. Therefore, it's necessary to establish mathematic model to research its features in three phases imbalance and put forward relevant control strategy to maintain its stable operation, which is of great positive significance. Reference [9 10] adopt the symmetrical component method to divide the original three-phases unbalance system into positive-sequence and negative- sequence parts and adopt the positive-sequence current controller base on the positive-sequence synchronization reference frame and the negative-sequence current controller based on the negative-sequence synchronization reference frame to control positive current and negative current respectively and independently. Reference [11] introduces internal model control to the PWM control of three-phase unbalance to realize isochronous control and to reduce the harmonic component. Reference [12] apply a predictive direct power control strategy in three-phase unbalance PWM control to avoid the separation between positive-sequence current and negative-sequence current. Reference [13] compensates pulsating power in case of grid's three-phase unbalance through a power compensator.

When the distributed generation selected as the master in the microgrid stop working, due to the loss of system voltage direction, the above mentioned strategy is no longer applicable. In order to maintain solid voltage transformer's stable operation in microgrid passive model when microgrid side three-phase unbalance occurs, this paper, based on detail analysis of SST mathematic mode under three-phase unbalance grid, puts forward a balance control strategy to restrain system three-phase unbalance. The simulation result shows that the control strategy could stably pass all active power and effectively prevent three-phase unbalance from entering into distribution grid side to improve system stability.

\section{THE CONFIGURATION OF SST}

SST (also called power electronic transformer, PET), by combining the power electronic technology and high frequency power transform technology to realize the transformation of electricity of different electric features. The configuration of SST is as indicated in Fig. 2.

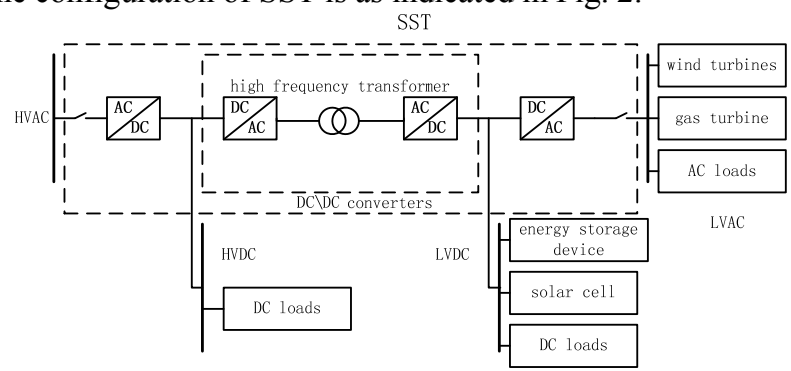

Figure 1. The configuration of SST 


\section{MATHEMATIC MODEL OF CONVERTER}

In case of three-phase unbalance, the three-phase converter's topological structure is as indicated in Fig.2.

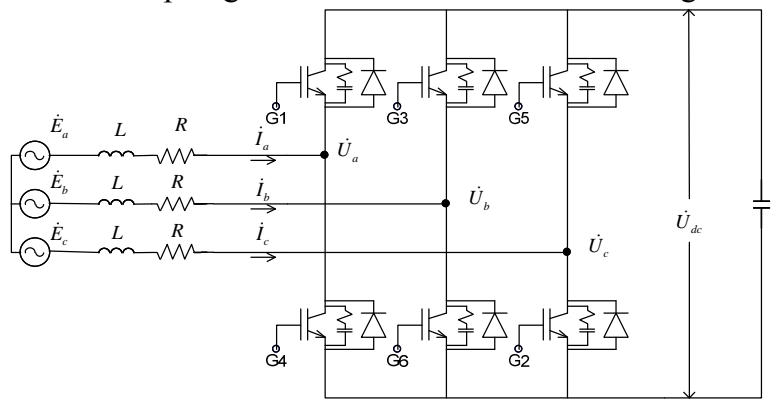

Figure 2. topological structure of converter

In the three phase non-earthed neutral line system, due to the shortage of zero-sequence current return circuit. The influence of zero-sequence component can be ignored. In order to provide convenience to analysis, higher harmonic component will be ignore and only positive-sequence and negative-sequence component will be taken into consideration. In case that the electricity loss in converter will be ignored, the power that pass converter is:

$$
\begin{aligned}
S= & P+j Q=E_{\alpha \beta} \cdot \bar{I}_{\alpha \beta} \\
= & \frac{\left[e^{\left(j \theta^{p}\right)}\left(E_{d}^{p}+j E_{q}^{p}\right)+e^{\left(-j \theta^{n}\right)}\left(E_{d}^{n}+j E_{q}^{n}\right)\right] .}{\left[e^{\left(j \theta^{p}\right)}\left(I_{d}^{p}+j I_{q}^{p}\right)+e^{\left(-j \theta^{n}\right)}\left(I_{d}^{n}+j I_{q}^{n}\right)\right]}
\end{aligned}
$$

The $\theta^{p}$ is the angle between the $\mathrm{d}$ axis in the positivesequence dq-coordinate system and $\alpha$-axis in the positivesequence $\alpha \beta$-coordinate system. The $\theta^{n}$ is the angle between the $\mathrm{d}$ axis in the negative-sequence dq-coordinate system and $\alpha$-axis in the negative-sequence $\alpha \beta$-coordinate system.

Through further derivation on Eq. (1):

$$
\left\{\begin{array}{c}
P=P_{0}+P_{c 2} \cos \left(\theta^{p}+\theta^{n}\right)+P_{s 2} \sin \left(\theta^{p}+\theta^{n}\right) \\
Q=Q_{0}+Q_{c 2} \cos \left(\theta^{p}+\theta^{n}\right)+Q_{s 2} \sin \left(\theta^{p}+\theta^{n}\right)
\end{array}\right.
$$

In Eq. (2)

$$
\left\{\begin{array}{c}
P_{0}=1.5\left(E_{\mathrm{d}}^{p} I_{\mathrm{d}}^{p}+E_{\mathrm{q}}^{p} I_{\mathrm{q}}^{p}+E_{\mathrm{d}}^{n} I_{\mathrm{d}}^{n}+E_{\mathrm{q}}^{n} I_{\mathrm{q}}^{n}\right) \\
P_{c 2}=1.5\left(E_{\mathrm{d}}^{p} I_{\mathrm{d}}^{n}+E_{\mathrm{q}}^{p} I_{\mathrm{q}}^{n}+E_{\mathrm{d}}^{n} I_{\mathrm{d}}^{p}+E_{\mathrm{q}}^{n} I_{\mathrm{q}}^{p}\right) \\
P_{\mathrm{s} 2}=1.5\left(E_{\mathrm{d}}^{p} I_{\mathrm{q}}^{n}-E_{\mathrm{q}}^{p} I_{\mathrm{d}}^{n}-E_{\mathrm{d}}^{n} I_{\mathrm{q}}^{p}+E_{\mathrm{q}}^{n} I_{\mathrm{d}}^{p}\right) \\
Q_{0}=1.5\left(-E_{\mathrm{d}}^{p} I_{\mathrm{q}}^{p}+E_{\mathrm{q}}^{p} I_{\mathrm{d}}^{p}-E_{\mathrm{d}}^{n} I_{\mathrm{q}}^{n}+E_{\mathrm{q}}^{n} I_{\mathrm{d}}^{n}\right) \\
Q_{c 2}=1.5\left(-E_{\mathrm{d}}^{p} I_{\mathrm{q}}^{n}+E_{\mathrm{q}}^{p} I_{\mathrm{d}}^{n}-E_{\mathrm{d}}^{n} I_{\mathrm{q}}^{p}+E_{\mathrm{q}}^{n} I_{\mathrm{d}}^{p}\right) \\
Q_{\mathrm{s} 2}=1.5\left(E_{\mathrm{d}}^{p} I_{\mathrm{d}}^{n}+E_{\mathrm{q}}^{p} I_{\mathrm{q}}^{n}-E_{\mathrm{d}}^{n} I_{\mathrm{d}}^{p}+E_{\mathrm{q}}^{n} I_{\mathrm{q}}^{p}\right)
\end{array}\right.
$$

Judged from Eq. (2), in case of three-phrase unbalance grid, the active power that pass through SST can be divided into DC component $P_{0}$, the cosine component of the second harmonic $P_{\mathrm{c} 2}$ and the sine component of the second harmonic $P_{\mathrm{s} 2}$. Reactive power can be divided into DC component $Q_{0}$, the cosine component of the second harmonic $Q_{\mathrm{c} 2}$ and the sine component of the second harmonic $Q_{\mathrm{s} 2}$.

In case that there is distributed generation working in the microgrid, since that voltage direction can be got from system, system's positive-sequence voltage component $E^{p}$ can be coincided with $d$ axis in the positive-sequence dqcoordinate system, while the negative-sequence voltage component $E^{n}$ can be coincided with the d axis in the negative-sequence dq-coordinate system. After the transformation, $E_{d}^{p}=E^{p}, E_{q}^{p}=0, E_{d}^{n}=E^{n}, E_{q}^{n}=0$. Therefore, the following result can be got from Eq. (2):

$$
\left[\begin{array}{l}
P_{0} \\
Q_{0} \\
P_{c 2} \\
P_{s 2}
\end{array}\right]=\frac{3}{2}\left[\begin{array}{cccc}
E^{p} & 0 & E^{n} & 0 \\
0 & -E^{p} & 0 & -E^{n} \\
E^{n} & 0 & E^{p} & 0 \\
0 & -E^{n} & 0 & E^{p}
\end{array}\right]\left[\begin{array}{c}
I_{d}^{p} \\
I_{q}^{p} \\
I_{d}^{n} \\
I_{q}^{n}
\end{array}\right]
$$

The reference value of $P_{0}$ can be got through a DC voltage-loop control; the reference value of $\mathrm{Q}_{0}$ can be get from an AC voltage-loop control, $P_{\mathrm{c} 2}=0, P_{\mathrm{s} 2}=0$. Judged from Eq. (3), when there are power sources working in the inverted side of microgrid, since all of the reference values of positive- sequence and negative-sequence current can be got, the current inner-loop control can be achieved. When the distributed generation selected as the master in the microgrid stop working and there is only load in the microgrid, due to the loss of system voltage direction, we need to consider changing control strategy.

\section{THTRR-PHASE UNBALANCE CONTROL STRAEGY OF SST UNDER PASSIVE MICROGRID}

Under passive three-phase unbalance microgrid, the inverter side of SST needs to achieve the following control targets: 1. Output voltage of inverter is three-phase balance and whose waveform is a sine curve; 2 . The amplitude and frequency of output voltage are constant and are able to effectively resist grid voltage fluctuation and load interruption; 3. In case of three-phase unbalance failure or three-phase load unbalance, it can effectively prevent threephase unbalance from entering into distribution grid side

In order to achieve control targets 1 and 2, it has to meet the condition of $E_{d}^{p}=E^{*}, E_{q}^{p}=0, E_{d}^{n}=0, E_{q}^{n}=0$. Meanwhile the d-axis of positive-sequence dq-coordinate system shall maintain $\theta_{0}^{p}$ when $t=0$; the rate of the $\mathrm{d}$ axis in the positivesequence in the speed of $\omega=2 \pi f_{n}$, while the negativesequence voltage component $E^{n}$ can be coincided with the d axis in the negative-sequence dq-coordinate system. Therefore, putting in Eq. (3) can get: 


$$
\left[\begin{array}{l}
P_{0} \\
Q_{0} \\
P_{c 2} \\
P_{s 2}
\end{array}\right]=\frac{3}{2}\left[\begin{array}{cccc}
E^{*} & 0 & 0 & 0 \\
0 & E^{*} & 0 & 0 \\
0 & 0 & E^{*} & 0 \\
0 & 0 & 0 & E^{*}
\end{array}\right]\left[\begin{array}{c}
I_{d}^{p} \\
I_{q}^{p} \\
I_{d}^{n} \\
I_{q}^{n}
\end{array}\right]
$$

In case of three-phase unbalance fault or three-phase unbalance load, equivalent resistance of the load is threephase unbalance; In case of the output voltage is three-phase balance, $I_{d}^{n}=I_{q}^{n}=0$ is false as well as $P_{\mathrm{c} 2}=0$ and $P_{\mathrm{s} 2}=0$ are false. The control target 3 cannot be fulfilled.

Based on above mentioned analysis, there is an inner contradiction among control targets 1,2 and 3 . The three control targets cannot be achieve at the same time. Therefore, the control requirements can be loosened to find other feasible solutions that allow output voltage to have unbalance voltage within restricted value and allow certain unbalanced component enter into distribution grid side, as indicated in Eq. (5).

$$
\left\{\begin{array}{l}
\left|E_{d}^{n^{*}}\right|<\varepsilon_{1} \\
\left|P_{c 2}^{*}\right|<\varepsilon_{2} \\
\left|P_{s 2}^{*}\right|<\varepsilon_{2}
\end{array}\right.
$$

Eq. (6) can be got through simple deduction:

$$
\left\{\begin{array}{l}
P_{c 2}^{*}=\min \left(\varepsilon_{2}, E_{\mathrm{d}}^{p^{*}} I_{d}^{n}+I_{d}^{p} \varepsilon_{1}\right) \\
P_{s 2}^{*}=\min \left(\varepsilon_{2}, E_{\mathrm{d}}^{p^{*}} I_{q}^{n}-I_{q}^{p} \varepsilon_{1}\right)
\end{array}\right.
$$

since we wish that the three-phase voltage in output side shall be balance as much as possible, $E_{d}^{p} \gg E_{d}^{n}$ is true. Therefore, putting into Eq. (3), it can be got:

$$
\left\{\begin{array}{l}
I_{d}^{n^{*}}=\frac{2 P_{c 2}^{*}}{3 E_{\mathrm{d}}^{p^{*}}} \\
I_{q}^{n^{*}}=\frac{2 P_{\mathrm{s} 2}^{*}}{3 E_{\mathrm{d}}^{p^{*}}}
\end{array}\right.
$$

From the Eq. (7) we can get the rated value for negativesequence current under unbalance grid. Meanwhile, the voltage-loop controller is introduced to maintain $E_{d}^{p}=E^{*}$ and $E_{q}^{p}=0$ for the sake of outputting constant three-phase sinusoidal voltage; both positive-sequence and negativesequence inner-loop controller are introduced respectively to make SST control current in accordance with the commands from voltage outer-loop and negative-sequence current calculator. The control strategy for three-phase unbalance in passive mode is indicated in Fig.3.

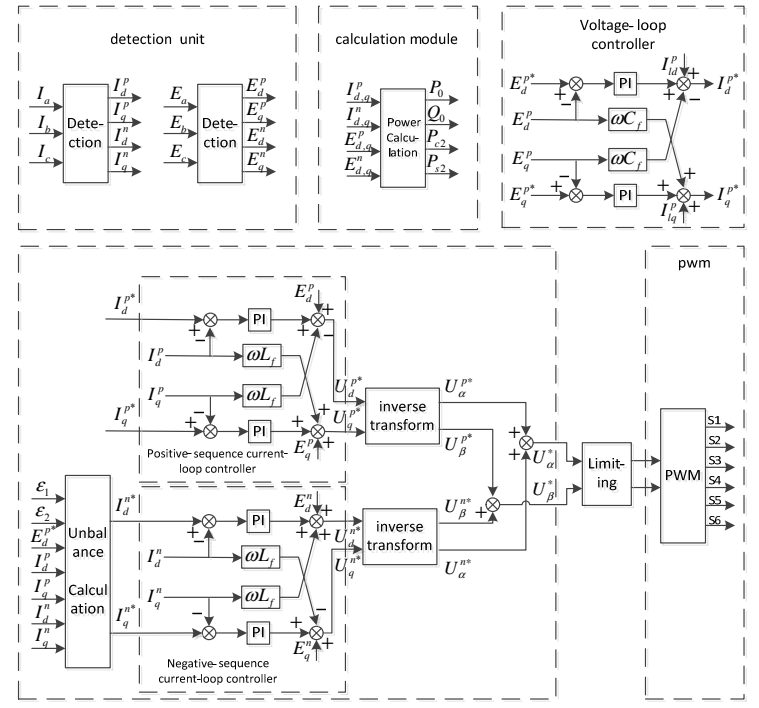

Figure 3. The control strategy for three-phase unbalance in passive mode

\section{SIMULATION AND RESULT ANALYSIS}

Simulation comparison is performed between the traditional voltage and current double-loop control strategy and the control strategy for three-phase unbalance in passive mode mentioned in this paper. The main simulation parameters are as indicated in table 1

TABLE I. SIMULATION PARAMETER

\begin{tabular}{|c|c|}
\hline Parameter & Value \\
\hline Grid Rated Voltage[kV] & 0.38 \\
\hline AC side Filter Inductor $[\mathrm{mH}]$ & 1.6 \\
\hline AC Side Filter Capacitor[mF] & 3 \\
\hline DC Side Voltage $[\mathrm{kV}]$ & 0.7 \\
\hline DC Side Capacitor $[\mathrm{mH}]$ & 50 \\
\hline Three Phase Equivalent Inductor[mH] & 0.1 \\
\hline Three Phase Equivalent Resistor $[\Omega]$ & 5 \\
\hline Failure Occurred Time[S] & 0.5 \\
\hline Failed Phase Load Equivalent Inductor[mH] & 0.05 \\
\hline Failed Phase Load Equivalent Resistor[ $\Omega]$ & 2.5 \\
\hline Fig 4 indicates the port Voltage & \\
\hline
\end{tabular}

Fig.4 indicates the port voltage information under the traditional voltage and current double-loop control strategy and the control strategy for three-phase unbalance in passive mode mentioned in this paper. Judged from the simulation curve, the traditional voltage and current double-loop control strategy can make the output voltage more balance than the control strategy for three-phase unbalance in passive mode. It's caused by the compromise on port voltage unbalance degree to maintain the consistency of active power, as indicated in Eq. (5). When degree of unbalance has exceeded the load's tolerance, the $\varepsilon_{1}$ can be reduced accordingly and the $\varepsilon_{2}$ can be increased to reduce port's unbalance to fulfill system's requirement. 


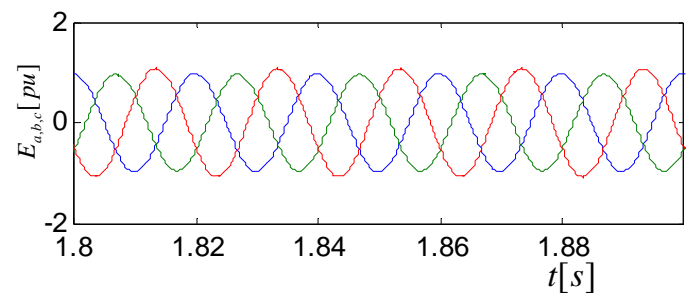

(a) The traditional double-loop control strategy

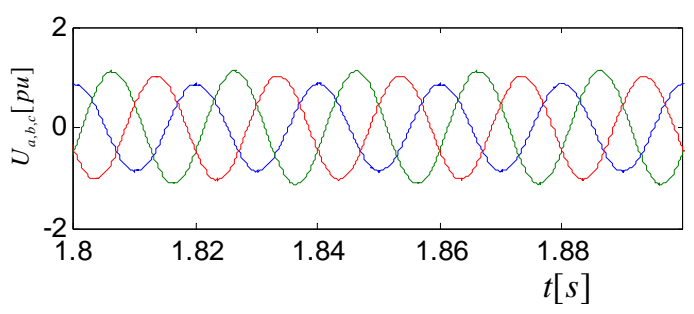

(b) The control strategy for three-phase unbalance

Figure 4. The port voltage information under two control strategies

Fig.5 reveals the active power status of the traditional voltage and current double-loop control strategy and the control strategy for three-phase unbalance in passive mode mentioned in this paper. The simulation curve proves that, under the circumstance of three-phase unbalance, the active power under the traditional voltage and current double-loop control strategy has significant fluctuation while the active power under the control strategy for three-phase unbalance in passive mode shows stabilization. Meanwhile, the active power's average value under the control strategy for threephase unbalance in passive mode mentioned by this paper is smaller than that under the traditional voltage and current double-loop control strategy The control strategy has successfully reduced the unbalanced phase's voltage rationally and has distributed the active power evenly, meanwhile, it reduce the current amplitude of unbalanced phase.

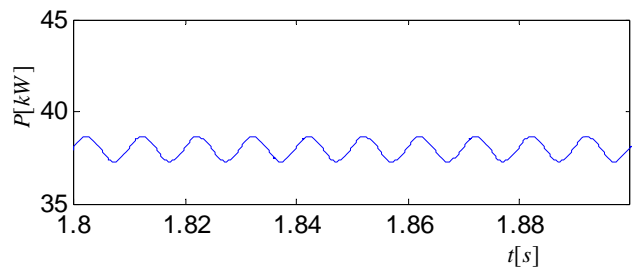

(a) The traditional double-loop control strategy

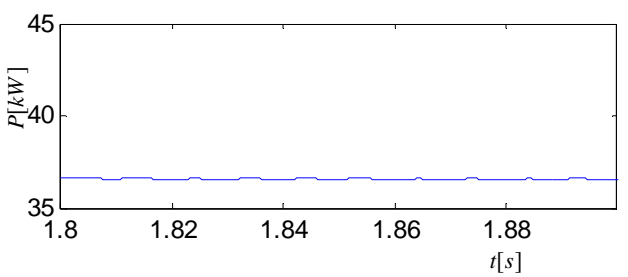

(b) The control strategy for three-phase unbalance

Figure 5. The active power status of under two control strategies

\section{ACKNOWLEDGMENT}

This paper, after analyzing SST mathematic model in detail, puts forward three-phase unbalance control strategy based on passive microgrid mode. The simulation comparison with the traditional voltage and current doubleloop control strategy prove that the control strategy is able to stabilize the active power through the SST and effective control the three-phase unbalance component inside microgrid to improve system stability. Since this paper doesn't make detailed restriction on grid unbalance and load unbalance during deduction and simulation, the three-phase unbalance controls strategy is applicable in different unbalance occasions. It's of certain significance to the researches on SST unbalance control. The simulation has proved the validity and efficiency of above mentioned strategy.

\section{REFERENCES}

[1] Lu Zongxiang,Wang Caixia,Min Yong, Overview on Microgrid Research[J], Automation of Electric Power Systems, 2007, 31(19): 100-107.

[2] Kundu, Debabrata, An overview of the distributed generation(DG) connected to the grid, 2008 Joint International Conference on Power System Technology POWERCON and IEEE Power India Conference, POWERCON 2008, 2008.

[3] Huang, Alex, FREEDM system - A vision for the future grid, IEEE PES General Meeting, PES 2010, 2010.

[4] Huang, Alex, FREEDM System: Role of power electronics and power semiconductors in developing an energy internet, Proceedings of the International Symposium on Power Semiconductor Devices and ICs, p9-12, 2009.

[5] Baran, Mesut, Smart distribution system research and education at the FREEDM systems center, IEEE Power and Energy Society General Meeting, 2011.

[6] She, $\mathrm{Xu}$, Performance evaluation of solid state transformer based microgrid in FREEDM systems, Conference Proceedings-IEEE Applied Power Electronics Conference and Exposition-APEC, p182188,2011

[7] She, Xu, Review of solid state transformer in the distribution system: From components to field application, 2012 IEEE Energy Conversion Congress and Exposition, ECCE 2012, p 4077-4084, 2012.

[8] Wang, Zhenyuan, Solid state transformer specification via feeder modeling and simulation, IEEE Power and Energy Society General Meeting, 2012.

[9] Rioual, Pascal, Regulation of a PWM rectifier in the unbalanced network state using a generalized model[J], IEEE Transactions on Power Electronics, v 11, n 3, p 495-502, May 1996.

[10] Song, Hong-seok, Dual current control scheme for PWM converter under unbalanced input voltage conditions[J], IEEE Transactions on Industrial Electronics, v 46, n 5, p 953-959, 1999.

[11] Zhang, Xing, Study of internal model control based three-phase PWM rectifier under unbalanced input voltage condition $[\mathrm{J}]$, Zhongguo Dianji Gongcheng Xuebao/Proceedings of the Chinese Society of Electrical Engineering, v 25, n 13, p 51-56, July 1, 2005

[12] Li, Zixin, Control of three-phase boost-type PWM rectifier in stationary frame under unbalanced input voltage[J], IEEE Transactions on Power Electronics, v 25, n 10, p 2521-2530, 2010.

[13] Wang, Meng, A power resonance compensation control strategy for PWM rectifiers under unbalanced grid voltage conditions[J], Zhongguo Dianji Gongcheng Xuebao/Proceedings of the Chinese Society of Electrical Engineering, v 32, n 21, p 46-53, July 25, 2012. 